\title{
A NOTE ON “OSCILLATION CRITERIA FOR SECOND-ORDER NONLINEAR NEUTRAL DYNAMIC EQUATIONS ON TIME SCALES"
}

\author{
HASSAN AHMED HASSAN AGWA, AHMED MOHAMED MOHAMED KHODIER \\ AND MAHMOUD HAMAM OSMAN SALM
}

\begin{abstract}
In this work, we give a counter example for the main result of the paper [Tamkang J. of Math., 43 (1)(2012), 109-122.] and we give the correct formula for some theorems given in their work.
\end{abstract}

\section{Introduction}

E. Thandapani and V. Piramanantham [1] considered the oscillation of the second order nonlinear neutral delay dynamic equation.

$$
\left(r(t)\left((y(t)+p(t) y(t-\tau))^{\Delta}\right)^{\gamma}\right)^{\Delta}+q(t) y^{\beta}(t-\delta)=0, t \in \mathbb{T},
$$

where $\mathbb{T}$ is a time scale.

They considered the following conditions:

$\left(H_{1}\right) \gamma \geq 1$ and $\beta \geq 1$ are quotients of odd positive integers.

$\left(H_{2}\right) \tau, \delta$ are nonnegative constants such that the delay functions $\tau: \mathbb{T} \rightarrow \mathbb{T}$ and $\delta: \mathbb{T} \rightarrow \mathbb{T}$ defined by $\tau(t)=t-\tau$ and $\delta(t)=t-\delta$.

$\left(H_{3}\right) \quad q(t)$ and $r(t)$ are real valued rd-continuous positive functions defined on $\mathbb{T}$. Also, $r(t)$ is a non decreasing function.

$\left(H_{4}\right) \quad p(t)$ is a positive and rd-continuous function on $\mathbb{T}$ such that $0 \leq p(t)<1$.

The authors in [1] obtained the oscillation criteria for Eq. (1.1) when

$$
\int_{t_{0}}^{\infty} \frac{\Delta(t)}{r^{\frac{1}{\gamma}}(t)}=\infty,
$$

Received October 28, 2014, accepted July 21, 2015.

2010 Mathematics Subject Classification. 34K11, 39A10, 39A99.

Key words and phrases. Oscillation, neutral equation, delay equation, dynamic equation, time scales. Corresponding author: Hassan Ahmed Hassan Agwa. 
or,

$$
\int_{t_{0}}^{\infty} \frac{\Delta(t)}{r^{\frac{1}{\gamma}}(t)}<\infty
$$

The main result for the oscillation of Eq. (1.1) in [1] is presented in the following theorem.

Theorem 1.1. Assume that condition (1.2) holds and there exist positive rd-continuous delta differentiable functions $\alpha(t)$ and $\phi(t)$ such that for every $b \geq 1$ and a positive number $M$, if

$$
\lim _{t \rightarrow \infty} \sup \int_{t_{0}}^{t}\left[\alpha(s) \phi(s) Q(s)-\frac{K(s) C^{2}(s)}{4 \phi(s) \beta M^{\frac{\gamma-1}{\gamma}} \alpha(s)}\right] \Delta s=\infty,
$$

where

$$
\begin{aligned}
Q(s) & =q(s)(1-p(s-\delta))^{\beta}, C(s)=\frac{\phi(s)\left(\alpha^{\Delta}(s)\right)_{+}}{\alpha^{\sigma}(s)}+\left(\phi^{\Delta}(s)\right)_{+}, \\
K(s) & =(b \cdot(s-\delta))^{1-\beta}\left(\alpha^{\sigma}(s)\right)^{2} r^{\frac{1}{\gamma}}(s-\delta), \\
\left(\alpha^{\Delta}(t)\right)_{+} & =\max \left\{\alpha^{\Delta}(t), 0\right\} \quad \operatorname{and}\left(\phi^{\Delta}(t)\right)_{+}=\max \left\{\phi^{\Delta}(t), 0\right\},
\end{aligned}
$$

then every solution of Eq. (1.1) is oscillatory on $\left[t_{0}, \infty\right) \mathbb{t}$.

We found that the conclusion of this theorem is not true. To illustrate it, we give the following counter example.

\section{Counter example}

Consider the second order delay differential equation.

$$
\left(t^{4}\left(x^{\prime}(t)\right)^{5}\right)^{\prime}+\left(\frac{1}{6}\right)^{6} \frac{1}{t^{\frac{7}{6}}(t-1)^{\frac{5}{6}}} x^{5}(t-1)=0, \mathrm{t}>1 .
$$

Here, We have $\quad \gamma=\beta=5, r(t)=t^{4}, p(t)=0$ and $\delta=1$. If we choose $\alpha(t)=1$ and $\phi(t)=t$, then by the Theorem (1.1), Eq.(2.1) is oscillatory. However, $x(t)=t^{\frac{1}{6}}$ is a positive solution of Eq.(2.1). Therefore Theorem (1.1) is not true. Tracing the error to it's source, we find that the main reason of this wrong is the use of the following false inequality in the proof of Theorem (1.1), they used

$$
\text { if } x(t) \leq b t \text {, then } x^{1-\beta}(t-\delta) \leq b^{1-\beta}(t-\delta)^{1-\beta} \quad \text { for } \beta \geq 1, t \in\left[T_{1}, \infty\right)_{\mathbb{T}} .
$$

\section{The correct formula for some results of [1]}

In the following, we give the correct formula for some theorems given in [1]. The correct formula of Theorem (1.1) in [1]. 
Theorem 3.1. Assume that $\left(H_{1}\right)-\left(H_{4}\right)$ hold, condition (1.2) holds and $\gamma \geq \beta \geq 1$. Also, assume that there exist positive rd-continuous delta differentiable functions $\alpha(t)$ and $\phi(t)$ such that for every $b \geq 1$ and a positive number $k \in(0,1)$, if

where

$$
\lim _{t \rightarrow \infty} \sup \int_{t_{0}}^{t}\left[\alpha(s) \phi(s) Q(s)-\frac{C^{2}(s)}{4 \phi(s) F(s)}\right] \Delta s=\infty,
$$

$$
\begin{aligned}
Q(s) & =q(s)(1-p(s-\delta))^{\beta}, C(s)=\frac{\phi(s)\left(\alpha^{\Delta}(s)\right)_{+}}{\alpha^{\sigma}(s)}+\left(\phi^{\Delta}(s)\right)_{+}, \\
F(s) & =\frac{\beta k^{\beta-1} \delta^{\beta-1}(s) \alpha(s) r^{\frac{1-\gamma}{\gamma}}(\sigma(s))}{2^{\gamma-1} b^{\gamma-\beta}\left(\alpha^{\sigma}(s)\right)^{2} r^{\frac{1}{\gamma}}(\delta(s))}, \\
\left(\alpha^{\Delta}(t)\right)_{+} & =\max \left\{\alpha^{\Delta}(t), 0\right\} \text { and }\left(\phi^{\Delta}(t)\right)_{+}=\max \left\{\phi^{\Delta}(t), 0\right\},
\end{aligned}
$$

then every solution of Eq. (1.1) is oscillatory on $\left[t_{0}, \infty\right) \mathbb{t}$.

Proof. Suppose that $y(t)$ is a nonoscillatory solution of Eq. (1.1) and proceeding as in the proof of Theorem (2.2) [1] to get the inequality (2.8) which has the form

$$
w^{\Delta}(t) \leq-\alpha(t) Q(t)+\frac{\left(\alpha^{\Delta}(t)\right)_{+}}{\alpha^{\sigma}(t)} w^{\sigma}(t)-\frac{\beta \alpha(t) r^{\frac{1-\gamma}{\gamma}}(\sigma(t))}{r^{\frac{1}{\gamma}}(\delta(t))} \cdot \frac{\left(w^{\sigma}(t)\right)^{2}}{\left(\alpha^{\sigma}(t)\right)^{2}} \cdot \frac{x^{\beta-1}(\delta(t))}{\left(x^{\Delta}(\sigma(t))\right)^{\gamma-1}} .
$$

Now, we find an estimation for $\frac{-x^{\beta-1}(\delta(t))}{\left(x^{\Delta}(\sigma(t))\right)^{\gamma-1}}$.

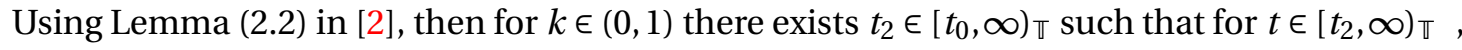
we have

$$
\begin{aligned}
x(\delta(t)) & \geq \frac{K \delta(t)}{\sigma(t)} x(\sigma(t)), \\
-x^{\beta-1}(\delta(t)) & \leq \frac{-K^{\beta-1} \delta^{\beta-1}(t) x^{\beta-1}(\sigma(t))}{\sigma^{\beta-1}(t)} .
\end{aligned}
$$

Hence,

$$
\frac{-x^{\beta-1}(\delta(t))}{\left(x^{\Delta}(\sigma(t))\right)^{\gamma-1}} \leq \frac{-K^{\beta-1} \delta^{\beta-1}(t) x^{\beta-1}(\sigma(t))}{\sigma^{\beta-1}(t)\left(x^{\Delta}(\sigma(t))\right)^{\gamma-1}} .
$$

Using Remark (2.1) in [2], then there exists $t_{3} \in\left[t_{0}, \infty\right)_{\mathbb{W}}$ such that for $t \in\left[t_{3}, \infty\right)_{\mathbb{T}}$, we have

$$
\begin{aligned}
\frac{x^{\Delta}(t)}{x(t)} & <\frac{2}{t}, \\
\frac{x^{\Delta}(\sigma(t))}{x(\sigma(t))} & <\frac{2}{\sigma(t)},
\end{aligned}
$$

hence, we have

$$
\begin{aligned}
&\left(x^{\Delta}(\sigma(t))\right)^{\gamma-1}<\frac{2^{\gamma-1}\left(x^{\gamma-1}(\sigma(t))\right)}{\sigma^{\gamma-1}(t)}, \\
& \frac{-1}{\left(x^{\Delta}(\sigma(t))\right)^{\gamma-1}}<\frac{-\sigma^{\gamma-1}(t)}{2^{\gamma-1}\left(x^{\gamma-1}(\sigma(t))\right)}
\end{aligned}
$$


Taking $t_{4}=\max \left\{t_{1}, t_{2}, t_{3}\right\}$ and substituting from (3.4) in (3.3), we get

$$
\frac{-x^{\beta-1}(\delta(t))}{\left(x^{\Delta}(\sigma(t))\right)^{\gamma-1}}<\frac{-K^{\beta-1} \delta^{\beta-1}(t)}{\sigma^{\beta-1}(t)} \cdot\left(\frac{\sigma(t)}{2}\right)^{\gamma-1} \cdot \frac{1}{x^{\gamma-\beta}(\sigma(t))} \text { for } t \in\left[t_{4}, \infty\right) \mathbb{T} .
$$

Since $x^{\Delta \Delta}<0$, then we have

$$
x(t)-x\left(t_{0}\right)=\int_{t_{0}}^{t} x^{\Delta}(s) \Delta s \leq x^{\Delta}\left(t_{0}\right)\left(t-t_{0}\right) .
$$

Therefore, there exist a $T_{1} \in\left[t_{0}, \infty\right)_{\mathbb{I}}$ and a suitable constant $b \geq 1$ such that for $t \in\left[T_{1}, \infty\right)_{\mathbb{T}}$ we have

$$
\begin{aligned}
x(t) & \leq b t, \\
x(\sigma(t)) & \leq b \sigma(t), \\
x^{\gamma-\beta}(\sigma(t)) & \leq b^{\gamma-\beta} \sigma^{\gamma-\beta}(t) .
\end{aligned}
$$

Hence,

$$
\frac{-1}{x^{\gamma-\beta}(\sigma(t))} \leq \frac{-1}{b^{\gamma-\beta} \sigma^{\gamma-\beta}(t)} .
$$

Taking $t_{5}=\max \left\{T_{1}, t_{4}\right\}$ and substituting from (3.6) in (3.5), we get

$$
\begin{aligned}
& \frac{-x^{\beta-1}(\delta(t))}{\left(x^{\Delta}(\sigma(t))\right)^{\gamma-1}}<\frac{-K^{\beta-1} \delta^{\beta-1}(t)}{\sigma^{\beta-1}(t)} \cdot\left(\frac{\sigma(t)}{2}\right)^{\gamma-1} \cdot \frac{1}{b^{\gamma-\beta} \sigma^{\gamma-\beta}(t)} \quad \text { for } t \in\left[t_{5}, \infty\right)_{\mathbb{T}}, \\
& \frac{-x^{\beta-1}(\delta(t))}{\left(x^{\Delta}(\sigma(t))\right)^{\gamma-1}}<\frac{-K^{\beta-1} \delta^{\beta-1}(t)}{2^{\gamma-1} b^{\gamma-\beta}} .
\end{aligned}
$$

Substituting from (3.7) in (3.2), we get

$$
w^{\Delta}(t) \leq-\alpha(t) Q(t)+\frac{\left(\alpha^{\Delta}(t)\right)_{+}}{\alpha^{\sigma}(t)} w^{\sigma}(t)-\frac{\beta \alpha(t) r^{\frac{1-\gamma}{\gamma}}(\sigma(t))}{r^{\frac{1}{\gamma}}(\delta(t))} \cdot \frac{\left(w^{\sigma}(t)\right)^{2}}{\left(\alpha^{\sigma}(t)\right)^{2}} \cdot \frac{K^{\beta-1} \delta^{\beta-1}(t)}{2^{\gamma-1} b^{\gamma-\beta}} .
$$

Multiply both sides of (3.8) by $\phi(t)$ and integrating from $t_{5}$ to $t$, we get

$$
\begin{aligned}
\int_{t_{5}}^{t} \alpha(s) \phi(s) Q(s) \Delta s \leq & -\int_{t_{5}}^{t} \phi(s) w^{\Delta}(s) \Delta s+\int_{t_{5}}^{t} \phi(s) \cdot \frac{\left(\alpha^{\Delta}(s)\right)_{+}}{\alpha^{\sigma}(s)} w^{\sigma}(s) \Delta s \\
& -\int_{t_{5}}^{t} \phi(s) \cdot \frac{\beta K^{\beta-1} \delta^{\beta-1}(s) \alpha(s) r^{\frac{1-\gamma}{\gamma}}(\sigma(s))}{2^{\gamma-1} b^{\gamma-\beta}\left(\alpha^{\sigma}(s)\right)^{2} r^{\frac{1}{\gamma}}(\delta(s))}\left(w^{\sigma}(s)\right)^{2} \Delta s .
\end{aligned}
$$

Using integration by parts, we get

$$
\begin{gathered}
-\int_{t_{5}}^{t} \phi(s) w^{\Delta}(s) \Delta s=-\phi(t) w(t)+\phi\left(t_{5}\right) w\left(t_{5}\right)+\int_{t_{5}}^{t} \phi^{\Delta}(s) w^{\sigma}(s) \Delta s \\
-\int_{t_{5}}^{t} \phi(s) w^{\Delta}(s) \Delta s<\phi\left(t_{5}\right) w\left(t_{5}\right)+\int_{t_{5}}^{t} \phi^{\Delta}(s) w^{\sigma}(s) \Delta s .
\end{gathered}
$$


Substituting from (3.11) in (3.9), we get

$$
\int_{t_{5}}^{t} \alpha(s) \phi(s) Q(s) \Delta s<\phi\left(t_{5}\right) w\left(t_{5}\right)+\int_{t_{5}}^{t} C(s) w^{\sigma}(s) \Delta s-\int_{t_{5}}^{t} \phi(s) F(s)\left(w^{\sigma}(s)\right)^{2} \Delta s .
$$

The above inequality can be written as:

$$
\int_{t_{5}}^{t} \alpha(s) \phi(s) Q(s) \Delta s<\phi\left(t_{5}\right) w\left(t_{5}\right)-\int_{t_{5}}^{t} \phi(s) F(s)\left(w^{\sigma}(s)-\frac{C(s)}{2 \phi(s) F(s)}\right)^{2} \Delta s+\int_{t_{5}}^{t} \frac{C^{2}(s)}{4 \phi(s) F(s)} \Delta s .
$$

Therefore,

$$
\begin{gathered}
\int_{t_{5}}^{t} \alpha(s) \phi(s) Q(s) \Delta s<\phi\left(t_{5}\right) w\left(t_{5}\right)+\int_{t_{5}}^{t} \frac{C^{2}(s)}{4 \phi(s) F(s)} \Delta s \\
\int_{t_{5}}^{t}\left[\alpha(s) \phi(s) Q(s) \Delta-\frac{C^{2}(s)}{4 \phi(s) F(s)}\right] \Delta s<\phi\left(t_{5}\right) w\left(t_{5}\right) .
\end{gathered}
$$

Taking the limit supremum of (3.15) as $t \rightarrow \infty$, we get a contradiction to condition (3.1). This completes the proof.

The correct formula of Theorem (2.9) in [1]:

Theorem 3.2. Assume that $\left(H_{1}\right)-\left(H_{4}\right)$ hold, condition (1.2) holds and $\gamma \geq \beta \geq 1$. Also, assume that there exist positive rd-continuous delta differentiable functions $\alpha(t)$ and $\phi(t)$ such that for every $b \geq 1$ and a positive number $k \in(0,1)$, if

$$
\begin{gathered}
\lim _{t \rightarrow \infty} \sup \frac{1}{H\left(t, t_{0}\right)} \int_{t_{0}}^{t}\left[\alpha(s) H(t, s) Q(s)-\frac{C^{2}(t, s)}{4 H(t, s) F(s)}\right] \Delta s=\infty, \\
Q(s)=q(s)(1-p(s-\delta))^{\beta}, \\
F(s)=\frac{\beta k^{\beta-1} \delta^{\beta-1}(s) \alpha(s) r^{\frac{1-\gamma}{\gamma}}(\sigma(s))}{2^{\gamma-1} b^{\gamma-\beta}\left(\alpha^{\sigma}(s)\right)^{2} r^{\frac{1}{\gamma}}(\delta(s))}, \\
C(t, s)=\frac{H(t, s)\left(\alpha^{\Delta}(s)\right)_{+}}{\alpha^{\sigma}(s)}+H^{\Delta_{s}}(t, s),
\end{gathered}
$$

then every solution of Eq. (1.1) is oscillatory on $\left[t_{0}, \infty\right) \mathbb{t}$.

Proof. Similar to that of Theorem (3.1).

\section{References}

[1] E. Thandapani and V. Piramanantham, Oscillation criteria for second order nonlinear neutral dynamic equations on time scales, Tamkang J. of Math., 43 (2012), 109-122.

[2] S.-Y. Zhang and Q.-R. Wang, Oscillation of second-order nonlinear neutral dynamic equations on time scales, Applied Math. and Computation, 216 (2010), 2837-2848. 
Department of Mathematics, Faculty of Education, Ain Shams University, Roxy, Cairo, Egypt.

E-mail: hassanagwa@yahoo.com

E-mail: khodier55@yahoo.com

E-mail: mahmoudhamam@edu.asu.edu.eg 